\title{
A Novel Solar Glass Window with Photovoltaic Power Generation and Automatic Temperature Regulation and Dust Removal Functions
}

\author{
Teng Zhang ${ }^{1}$, Jinpeng Wang ${ }^{1, *}$, Yang Zhou ${ }^{1}$, Xin Guan ${ }^{1}$, Wei Li $^{1}$, Xin Zhao ${ }^{1}$ \\ ${ }^{1}$ Dalian Polytechnic University, Dalian 116034, China \\ wangjpedlpu.edu.cn
}

\begin{abstract}
Solar energy is a new clean energy with many advantages compared with traditional energy, and the range of its application is becoming wider. This paper aims to combine the power technology based on solar energy with windows, so as to alleviate stuffiness indoors in summer and save energy. For this purpose, a novel solar glass window is designed, which reduces indoor temperature through air flow, a solar energy panel is installed in the window frame to provide power to the indoor air conditioner used for auxiliary cooling, and the air-conditioning switch is automatically controlled according to room temperature, dust on the solar energy panel is removed according to dust deposition, so as to improve the photoelectric conversion efficiency.
\end{abstract}

Keywords: Photovoltaic glass, Solar power supply, Intelligent temperature control, Automatic dust removal

\section{Introduction}

In society today, energy has become a hot topic. Fossil fuel, as a traditional non-renewable energy source, is still one of the major energies on which we depend. Its excessive consumption not only accelerates the depletion of non-renewable energy, but also aggravates environmental pollution. Nevertheless, building energy is an important portion of total energy, accounting for about $40 \%$ [1], which consumes a large part of fossil energy. This problem shall never be ignored.

Compared with non-renewable energy, solar energy is advantageously characterized in pollution free, large reserve and long-lasting usage, etc. and a main way of generating power from solar energy is photovoltaic (PV) power generation. By combining windows with PV power generation, part of the energy crisis can be alleviated. In Japan, the government implemented "Sunshine Project" and by the same built several typical solar heating and air conditioner test buildings, such as Yazaki test solar house [2]. Kai $\mathrm{Li}$ established the overall relative power loss model of non-uniform width bus grid solar cells and found that when the number of bus grid lines is 2-6, compared with the typical constant width grid line structure solar cells, the photovoltaic Conversion efficiency increased by $0.10 \%, 0.09 \%, 0.10 \%, 0.09 \%$ and $0.09 \%$ respectively [3]. Sonveer carried out an optimized analysis on the 
thermoelectric properties of translucent PV glass with hybrid double channels by using the fuzzy genetic algorithm, the power generation efficiency was improved by $12.21 \%$, and the mean temperature of PV glass was decreased by $2.28^{\circ} \mathrm{C}$ [4]. Antika team analyzed the relationship between temperature and power generation efficiency by combining experiments and models [5]. Ye Huang built an experimental platform for the Concentrated Photovoltaic Photothermal (CPV/T) system using dual-axis tracking, and found that the average photoelectric conversion efficiency of the system reached $13.0 \%$ [6]. Through experiment, Xiaoli WU analyzed the influence of the temperature of hollow PV glass component on its electrical property. The results indicated that the negative effect of temperature increase on conversion rate shall be taken into consideration when the outdoor temperature is high [7].

An important parameter which can denote photovoltaic power generation is the photoelectric conversion rate. The papers by the above and others has contributed to the field of using sunlight for power generation, and different methods have been used to improve the photoelectric conversion rate. However, these papers did not consider the form of photovoltaic power generation combining multiple functions, and did not provide an exact solution for dust, an important factor affecting the photoelectric conversion rate. Compared with uncomplicated power generation function, the novel solar glass window in this paper realizes intelligent temperature control while supplying power to indoor air conditioner, and automatically control the turn-on and turn-off of cooling equipment according to the indoor temperature. However, the dust accumulated on solar energy panel can reflect, scatter and absorb solar radiation to decrease the transmittance of solar radiation, so the output power decreases accordingly, which severely affects its photoelectric conversion efficiency. In order to solve the problem of low photoelectric conversion efficiency due to dust accumulation, this solar glass window is equipped with photoelectric sensor and dust removal device, which enables functions of automatic dust removal according to the dust accumulation on the solar energy panel.

\section{System overview}

This design first installs the suitable size solar panel on the window frame, absorbs the solar energy to supply the indoor power. Different from the single-layer glass used for Windows of most buildings, this design adopts double-layer glass structure, and combines the flexible thin film solar cells with semi-transparent nature on the outer glass [8-9]. It is also used to generate electricity for indoor air conditioning without affecting indoor lighting requirements. Some small holes are drilled in the upper and lower parts respectively to facilitate air flow in and out. The inner glass is made of ordinary glass. Photoelectric sensor and dust removal device are installed on the outside of the window frame. According to the degree of ash accumulation, the photoelectric sensor sends electrical signals to the MSP430 single-chip microcomputer after amplification and shaping, this single chip microcomputer controls the operation of dust removal device to realize automatic dust removal. The 
elevation view and side view profile of the solar glass window are shown in Fig. 1. and Fig. 2. respectively.

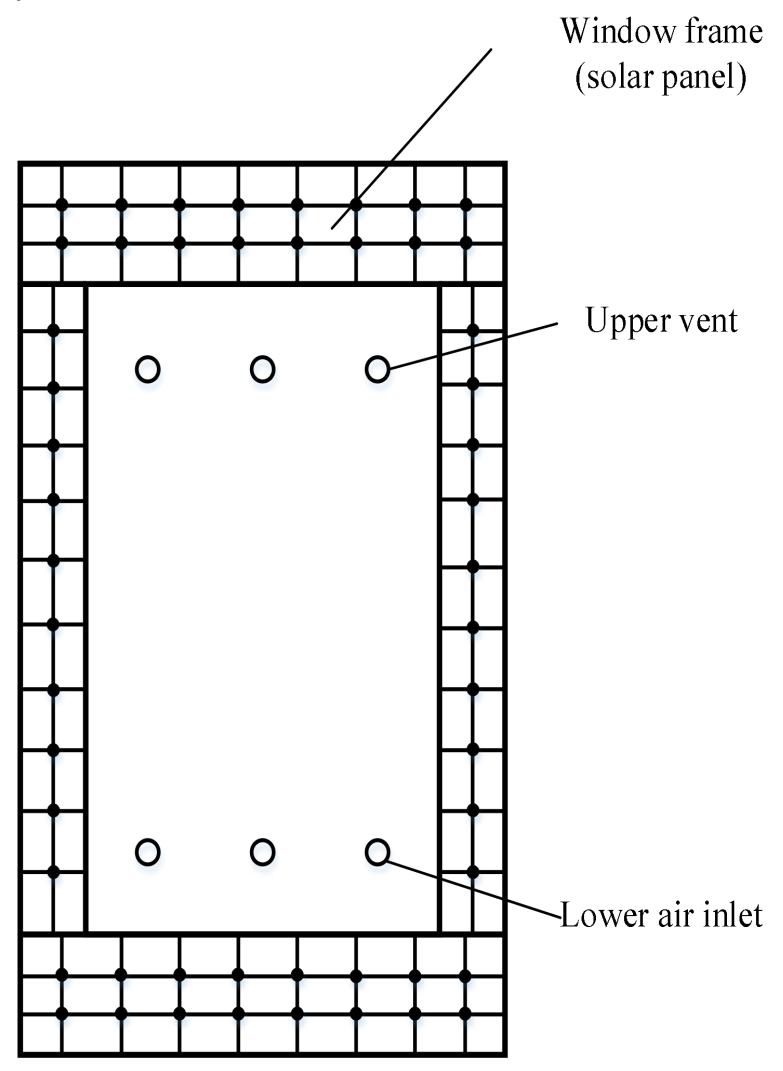

Fig. 1. Elevation view 


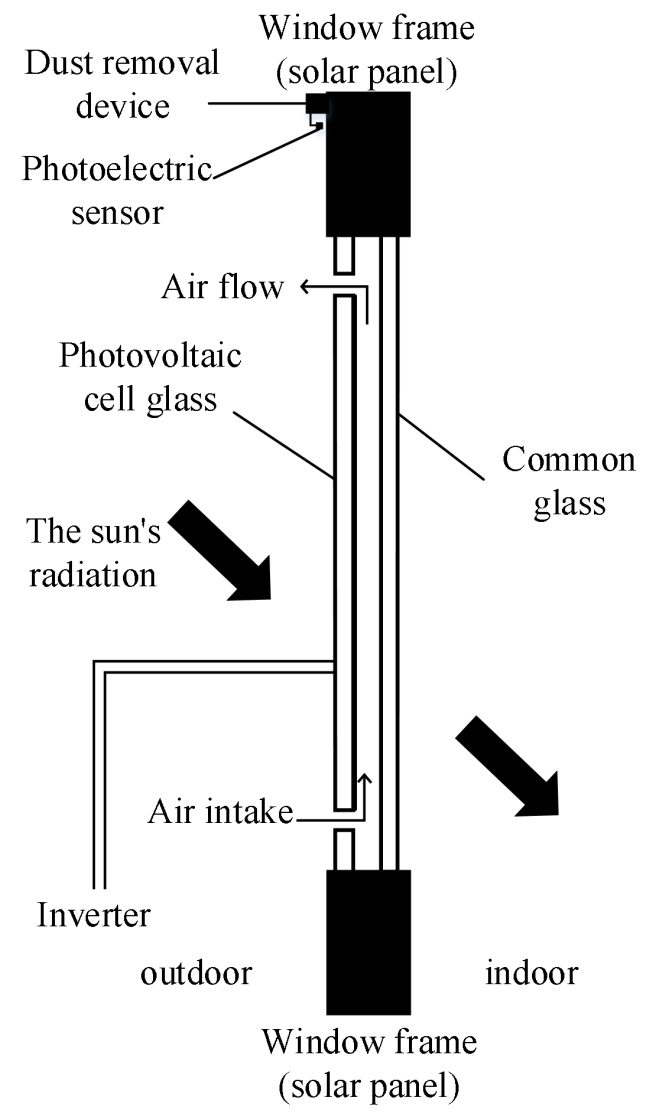

Fig. 2. Side view profile

\section{Functional principle}

\subsection{Reduce the room temperature}

The outdoor air enters the area between the two layers of glass through the air inlet under the outer glass. When the indoor temperature is too high, the indoor heat will be transferred to the air in the middle area through the ordinary glass in the inner layer, a flexible thin-film solar cell attached to the outer glass also generates heat from the sun, which is also transferred to the air in the middle layer, the heated air becomes denser and rises, passing out through the upper vent of the outer glass, in a process that reduces the room's temperature. 


\subsection{Photovoltaic power generation}

The composition of photovoltaic power generation system needs to take into account generation, storage, transmission and automatic control. In the new solar glass window designed in this paper, the power generation system is a flexible thin-film solar cell on the outer glass and a solar panel installed on the window frame. They can convert the solar energy shining on its surface into electricity for indoor cooling equipment, such as air conditioning, The storage system consists of a photovoltaic controller and a battery bank. The battery bank is composed of a single battery in series and in parallel. Its function is to store the electric energy converted into light energy for overall deployment, and the unit is relatively independent. The function of the photovoltaic controller is to control the charging process of the battery, let the photovoltaic array charge the battery, and carry out overcharge and overdischarge protection on the battery, so as to protect the normal use of the battery and ensure the normal operation of the system [10]. While the electricity converted from solar energy is direct current, the air conditioner is powered by alternating current. In order to make the air conditioner work normally, the inverter is needed to change the direct current in the battery into alternating current. In order to optimize the utilization of the whole photovoltaic power generation system, it is very important to calculate the conversion efficiency of the inverter and select the inverter with suitable specifications.

The conversion efficiency of the inverter is the ratio of output power to input power. The input power of the inverter is calculated as follows:

$$
P_{D C}=I_{D C} \times V_{D C}
$$

Among them, $P_{D C}$ is direct current power, $I_{D C}$ and $V_{D C}$ are DC current value and DC voltage value respectively, which are generated by photovoltaic cells. According to Kirchhoff's law (KCL), the current $I_{D C}$ generated by the photovoltaic cell can be obtained as:

$$
I_{D C}=I_{p h}-I_{D}-I_{s h}
$$

Among them, $I_{p h}$ is the photo-generated current and $I_{D}$ is the current generated by the $\mathrm{P}-\mathrm{N}$ junction of the photovoltaic cell itself, which can be further calculated to obtain:

$$
I_{D C}=I_{p h}-I_{o}\left[e^{\frac{q\left(V_{D C}+I_{D C} R_{s}\right)}{A k T}}-1\right]-\frac{V_{D C}+I_{D C} R_{s}}{R_{s h}}
$$

Among them, $I_{o}$ is the reverse saturation current, $q$ is the charge of electrons, $A$ is the quality factor of the diode, $T$ is the temperature of the photovoltaic cell, and $k$ is the Boltzmann constant, whose value is about $1.3806 \times 10^{-23} \mathrm{~J} / \mathrm{K}$.

In the case of an open circuit, due to the large resistance of the parallel parasitic resistance $R_{s h}$ in the photovoltaic cell, the open circuit voltage can be obtained as: 


$$
V_{O C} \approx \frac{A k T}{q} \ln \left(\frac{I_{p h}}{I_{0}}+1\right)
$$

In practical applications, $I_{s h}$ and $I_{D}$ are generally ignored, so that $I_{p h}$ is approximately equal to $I_{s c}$. In the case of an open circuit, the output voltage of the photovoltaic cell $V_{D C}=V_{O C}$, so:

$$
\begin{gathered}
I_{D C}=I_{s c}\left(1-C_{1}\left(e^{\frac{V_{D C}}{C_{2} V_{O C}}}-1\right)\right) \\
C_{1}=\left(1-\frac{I_{m}}{I_{s c}}\right) e^{\left(-\frac{V_{m}}{C_{2} V_{O C}}\right)} \\
C_{2}=\left(\frac{V_{m}}{V_{O C}}-1\right)\left[\ln \left(1-\frac{I_{m}}{I_{s c}}\right)\right]^{-1}
\end{gathered}
$$

Among them, $I_{m}$ and $V_{m}$ are the output current and output voltage of the photovoltaic cell operating at maximum power. The output voltage of the photovoltaic cell is affected by the sunlight intensity and the external environment temperature. Taking into account the changes of these two points, the temperature difference $\Delta T$ and the light intensity difference $\Delta S$ can be set as:

$$
\begin{aligned}
& \Delta T=T-T_{r e f} \\
& \Delta S=\frac{S}{S_{r e f}}-1
\end{aligned}
$$

Among them, $T_{\text {ref }}$ is the ambient temperature under standard conditions, which is $25^{\circ}$ C, and $S_{\text {ref }}$ is the sunlight intensity under standard conditions, which is $1000 \mathrm{~W} / \mathrm{m}^{2}$.

Finally, the values of $C_{l}$ and $C_{2}$ can be determined and obtained:

$$
\begin{gathered}
I_{D C}=I_{s c} \frac{S}{S_{r e f}}(1+a \Delta T) \\
V_{D C}=V_{O C}(1+c \Delta T \ln (1+b \Delta S))
\end{gathered}
$$

Among them, $a, b$, and $c$ are correction coefficients, and their values are $0.00225,0.5$, and 0.00288 , respectively [11].

The output power of the inverter can be calculated as follows:

$$
P_{A C}=I_{A C} \times V_{A C}
$$


Among them, $P_{A C}$ is $\mathrm{AC}$ electric power, $I_{A C}$ and $V_{A C}$ are $\mathrm{AC}$ current value and $\mathrm{AC}$ voltage value respectively, which are obtained by the inverter. Therefore, the conversion efficiency of the inverter can be calculated as follows:

$$
\eta=P_{A C} / P_{D C}
$$

\subsection{Intelligent temperature control}

When sunlight hits the outer glass attached to a flexible thin-film solar cell, the energy is divided into four parts. Some of the energy enters the room through the outer glass, Some of the energy is reflected back into the environment;Some of the energy is absorbed by photovoltaic cells and converted into electricity, The last part of the energy is converted into the thermal energy generated when the photovoltaic cell works [12]. The energy analysis on the outer glass is shown in Fig. 3.

Due to the inner layer of glass and the use of ordinary glass, through the outer layer of the sun to the inner glass on the surface of most of the energy will return through reflection and transmission effect to the surrounding environment, only a small percentage of the energy absorbed by photovoltaic cells and by convection and radiation heat transfer back to the surrounding environment, without considering the energy loss caused by reflection and transmission, the inner glass of heat exchange with the environment as shown in Fig. 4.

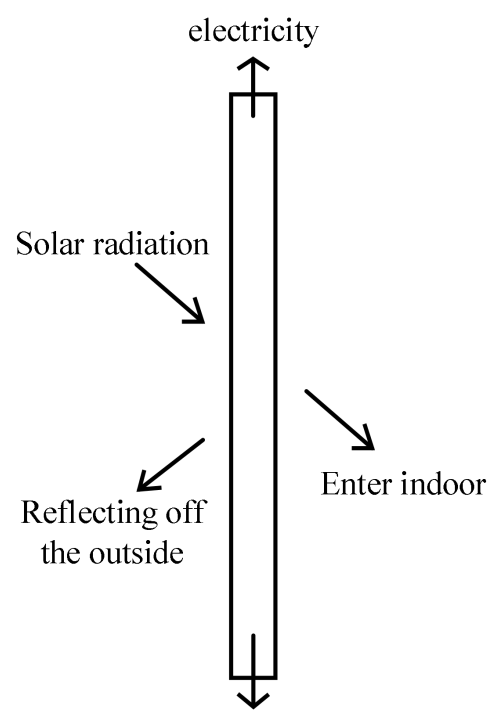

Heat energy

Fig. 3. Energy analysis diagram of outer glass 


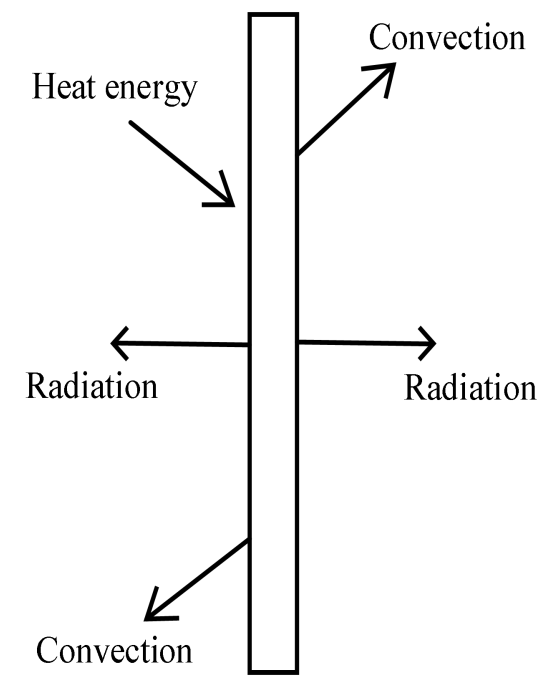

Fig. 4. Heat exchange diagram of inner glass with surrounding environment

The new type of solar glass window designed in this paper has limited ability to cool the indoor temperature through air circulation between two layers of glass. In hot summer, when the indoor temperature is too high, people need to turn on the air conditioner to help cool down. A temperature sensor is installed in the room to detect the indoor temperature. When the indoor temperature rises to a certain degree Celsius, the air conditioner can start itself to cool down the room, so as to realize the intelligent temperature regulation function of controlling the opening and closing of the air conditioner according to the room temperature. The principle of the Temperature sensor is that its resistance will decrease with the increase of Temperature, so the change of resistance value can show the change of indoor Temperature. The switch operation of the air conditioner can be controlled by the change of resistance value [13]. In this paper, the Negative Temperature Coefficient (NTC) thermistor is selected as the Temperature sensor of this system. Its resistance value is calculated as follows:

$$
R_{t}=\operatorname{Re}^{B\left(1 / T_{1}-1 / T_{2}\right)}
$$

In the formula, $R_{t}$ is the resistance value of thermistor at $T_{l}$ temperature, $R$ is the nominal resistance value of thermistor at $T_{2}$ room temperature, and $B$ is an important parameter of thermistor, The natural logarithm of both sides of Equation (14) is obtained:

$$
\ln R_{t}=\ln R+B\left(\frac{1}{T_{1}}-\frac{1}{T_{2}}\right)
$$


After transforming it, we get:

$$
\ln R_{t}=\frac{B}{T_{1}}+\left(\ln R-\frac{B}{T_{2}}\right)
$$

It can be seen that $\ln R_{t}$ has a linear relationship with $1 / T_{1}$, and the slope obtained by linear fitting is the $B$ value of the thermistor [14]. It reflects the sensitivity of the thermistor's resistance to changes in temperature, It is defined as the ratio of the difference between the natural logarithm of the zero-power resistance value at two temperatures and the difference between the reciprocal of the two temperatures. The formula is as follows:

$$
B=\frac{\ln \left(R_{T 1} / R_{T 2}\right)}{\left(1 / T_{1}\right)-\left(1 / T_{2}\right)}
$$

Among them, $R_{T 1}$ refers to the zero power resistance value at temperature $T_{1}$, and $R_{T 2}$ refers to the zero power resistance value at temperature $T_{2}$. Considering the accuracy of measurement problems in this system, 10k value of $3950 \mathrm{~B}$ is finally determined $\Omega$ thermistors.

However, in real life, people may be dissatisfied with the set temperature value that controls the opening of the air conditioner and choose to turn on the air conditioner manually. Therefore, manual mode is added in the process of controlling the air conditioner. On this basis, the timing mode of air conditioning operation time is added to make it more flexible and convenient. The circuit schematic diagram of using solar energy to supply power for air conditioning is shown in Fig. 5. 


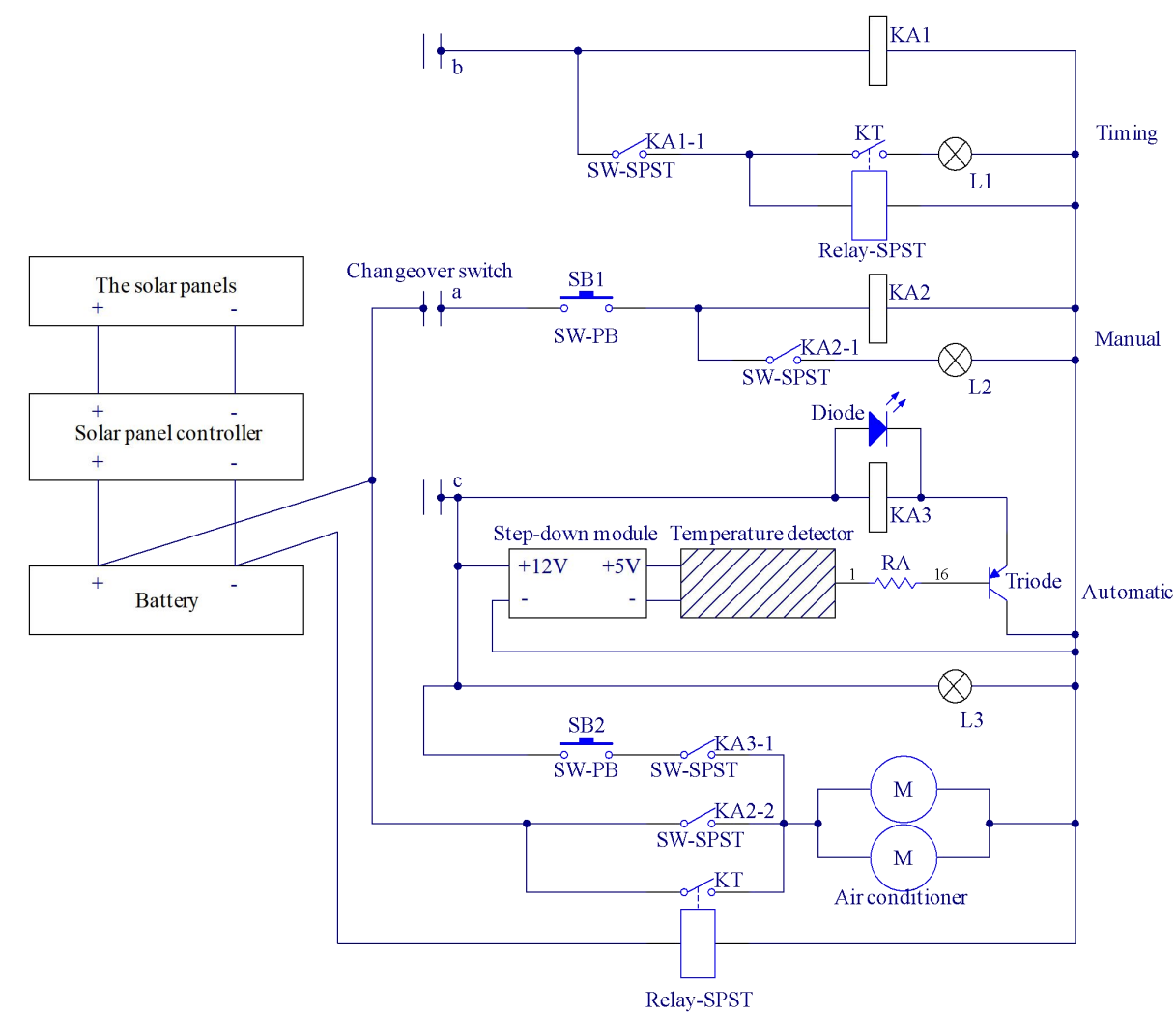

Fig. 5. Schematic diagram of intelligent temperature regulating circuit for solar power supply

\subsection{Automatic dust removal}

At present, the photoelectric conversion efficiency of solar cells is still not very ideal, usually only $10 \%$ to $20 \%$, and the conversion efficiency needs to be further improved. The accumulation of dust on the battery surface is an important factor affecting the photoelectric conversion efficiency. New solar glass window in the long-term use of the process will be affected by all kinds of weather factors, it is easy to form ash on its surface, especially in some harsh environment in the region, the speed of ash will be accelerated, whether the ash is timely treatment is a problem that cannot be ignored. The accumulated dust will not only reduce the daylighting rate of solar cell modules, affect their power generation efficiency, but also accelerate the aging speed of their internal structures, thus plunging the whole system into a vicious cycle [15].

The new solar glass window designed in this paper has an automatic dust removal function. The principle of the dust removal system is shown in Fig. 6. and the principle diagram of the circuit is shown in Fig. 7. 


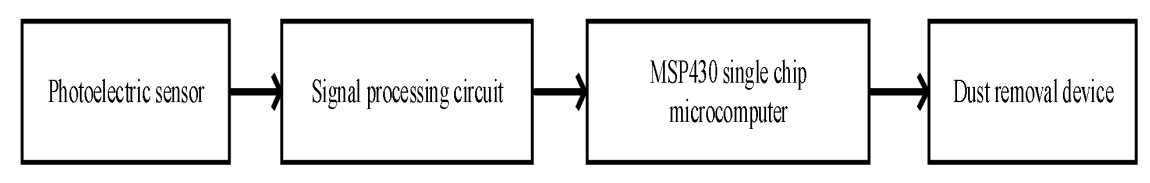

Fig. 6. Schematic diagram of automatic dust removal system

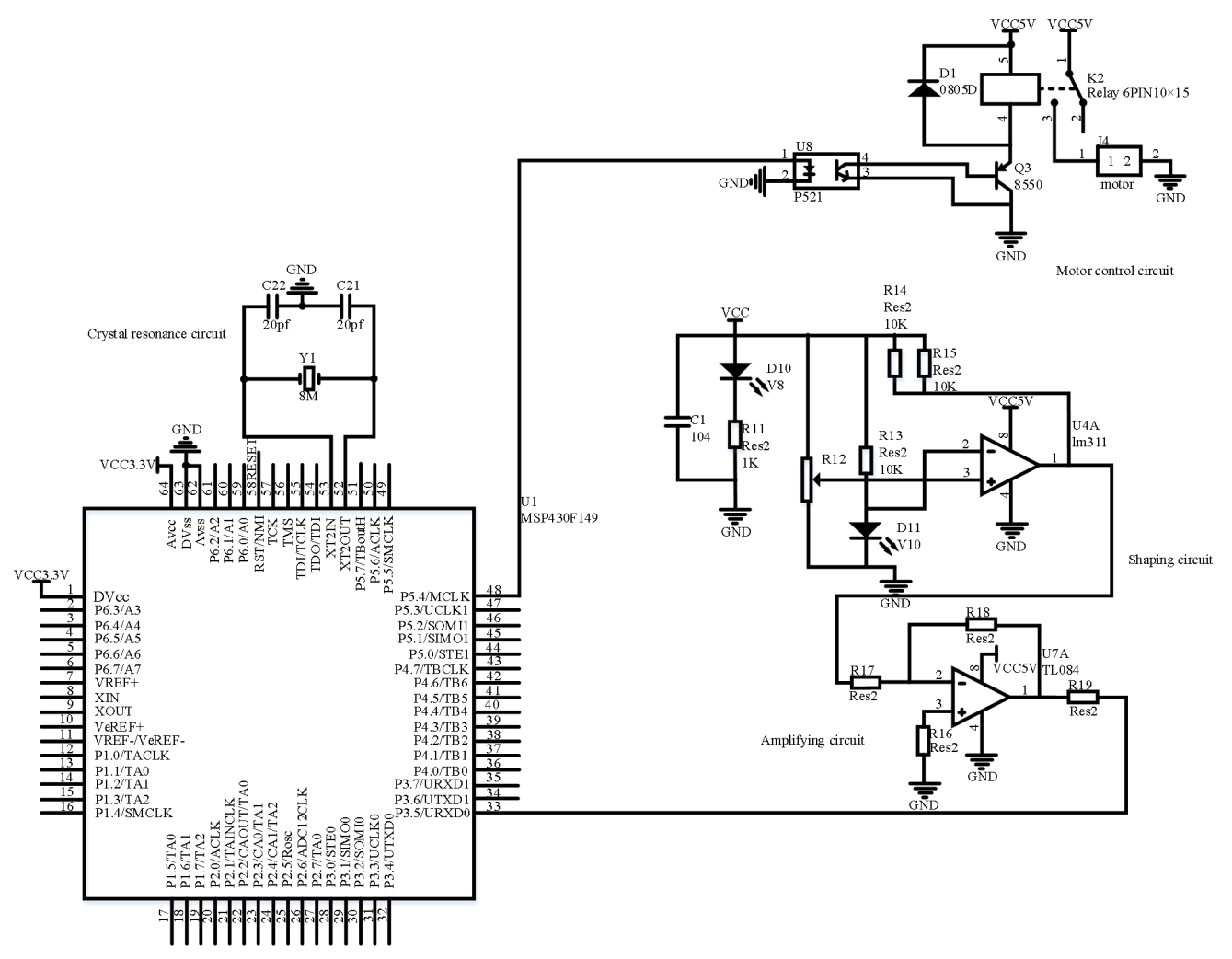

Fig. 7. Circuit schematic diagram of automatic dust removal system

Photoelectric sensor has the technical advantages of high resolution, high response speed and large information capacity, etc. In addition, its non-contact characteristics will not cause damage to the detection target and the sensor itself, which makes it can be used for a long time [16]. Because the photoelectric sensor has been working for a long time on the side outside the new glass window, it will be affected by some environmental factors. Therefore, the photoelectric sensor with higher sensitivity, lower energy consumption and easy maintenance should be selected. The photoelectric sensor selected by this system is E3F-DS30C4 adjustable diffuse reflection photoelectric switch, which is of NPN type with normally open structure. The detection distance is appropriate $(30 \mathrm{~cm})$, and the suitable ambient temperature 
range is wide $\left(-25^{\circ} \mathrm{C} \sim 70^{\circ} \mathrm{C}\right)$, meeting its due working conditions [17]. Photoelectric sensor in this system is the function of testing dust accumulation on photovoltaic modules, then send electrical signals to the system part of follow-up, when the dust on the solar panel is large, light irradiation to the solar panel is absorbed, and so cannot be reflected, the photoelectric switch into a closed state, sends out electric signals.

Because the electrical signal from the photoelectric sensor is very weak and unstable, it needs to be amplified and reshaped, so the signal processing circuit consists of operational amplifying circuit and reshaping circuit.The signal amplification circuit as shown in Fig. 8. is designed with TL084 as the operational amplifier. The TL084 chip has the advantages of small size, low power consumption, high conversion efficiency, low bias current and strong anti-interference [18].

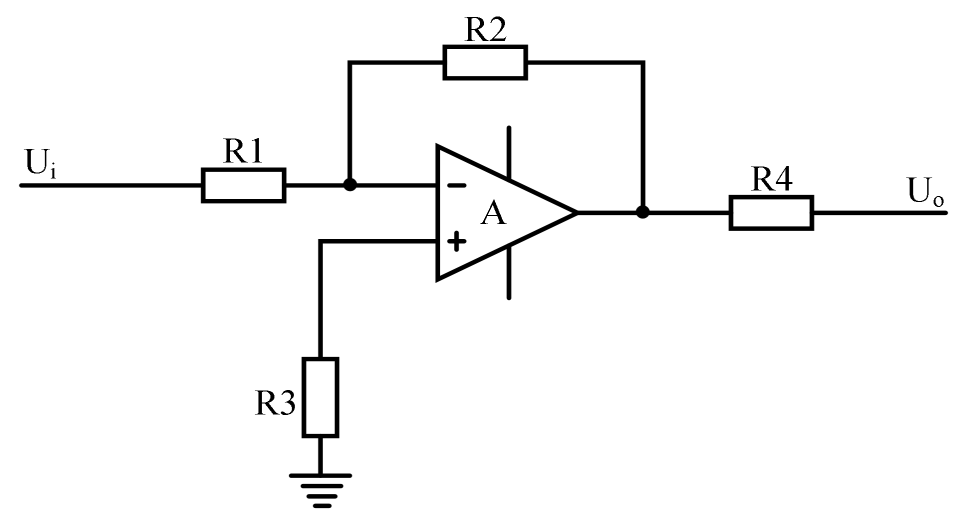

Fig. 8. Amplifying circuit schematic

The amplified signal is not a standard square wave and needs to go through a shaping circuit. Fig. 9. is a TTL shaping circuit with LM311 as the voltage comparator. The output pin of the amplifier is connected to an input pin of the voltage comparator, and the other input pin is connected to a potentiometer that can adjust the amplitude of the reference voltage. Adjusting the resistance value of $R_{t}$ can change the size of the reference voltage. Depending on the operating cost of photovoltaic power generation, different regions have different tolerances for dust accumulation, so the reference voltage will be different. 


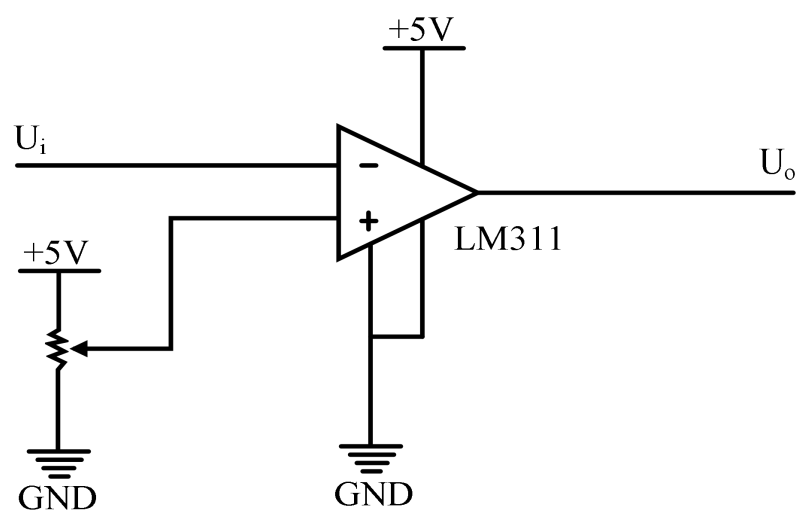

Fig. 9. TTL shaping circuit schematic diagram

After amplification and shaping, the signal is transmitted to the MSP340 MCU. The MSP430 MCU has its own ADC12 module, which is composed of a 12-bit SAR kernel, a sampling hold circuit, a reference voltage circuit and a clock circuit. With a low-power design, it only needs to set the corresponding register and power off independently to realize the conversion quickly and conveniently [19]. MSP430 microcontroller needs $3.3 \mathrm{~V}$ DC power supply to work, and the sensor and other peripheral circuits need $5 \mathrm{~V}$ power supply, so a $12-\mathrm{V}$ rechargeable battery can be used for power supply. The $2 \mathrm{~V}$ DC power supply is converted to a $5 \mathrm{~V}$ power supply through step-down switching voltage regulator LM2576 for peripheral circuits such as sensors and relays. The $5 \mathrm{~V}$ voltage is then converted to a $3.3 \mathrm{~V}$ voltage through voltage regulator LP2985 for use by MSP430 single chip microcomputer. The power supply circuit is shown in Fig. 10.

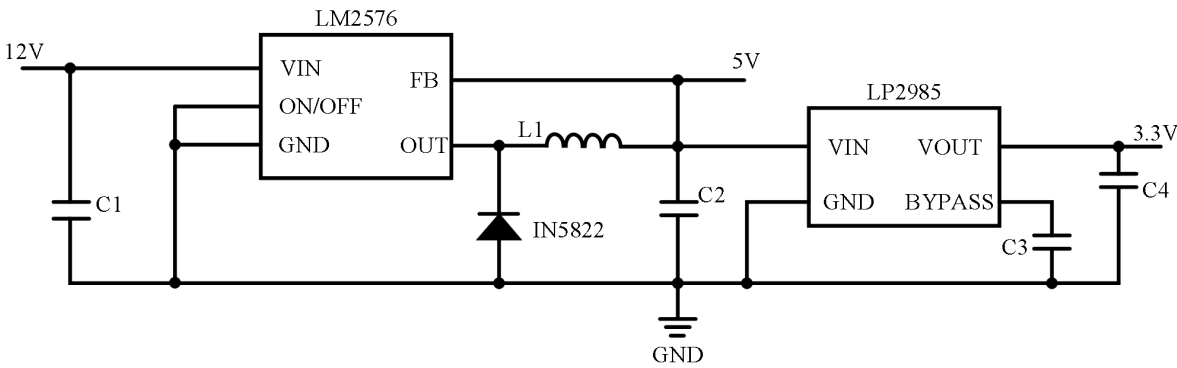

Fig. 10. Schematic diagram of power supply circuit

Since the MCU is a weak current system, it needs to be isolated from the strong current side to ensure safety and prevent the voltage backflow on the strong current side from burning out the MSP430. Therefore, the switch optocoupler is first used for photoelectric isolation, and then the relay is driven by the audion. The relay controls 
the motor in the dust removal device for dust removal, and the driving circuit is shown in Fig. 11.

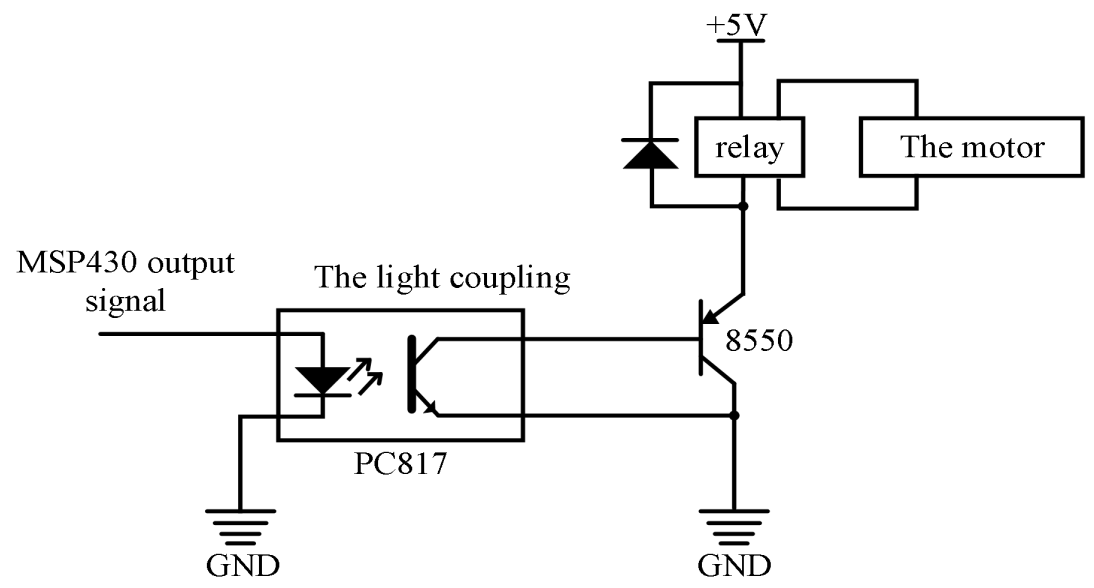

Fig. 11. Schematic diagram of drive circuit

The dust removal device is composed of brush head, DC motor and filter bag. The DC motor rotates to drive the brush head to work, breaking the binding force between dust and the surface of the solar panel, making the dust attached to the surface become suspended dust particles. Due to the high speed operation of the motor, the negative pressure difference is formed between the internal pressure of the main engine and the external atmosphere. Under the action of pressure difference, the suspended dust particles are inhaled into the filter bag, and the filter bag can be taken out for cleaning after being filled. Dust removal efficiency can be calculated by the following formula:

$$
\eta=\frac{C_{i n}-C_{o u t}}{C_{\text {in }}} \times 100 \%
$$

Among them, $C_{i n}$ is the dust mass concentration of the inlet flow and $C_{\text {out }}$ is the dust mass concentration of the outlet flow. The calculation expression of $C_{i n}$ is as follows:

$$
C_{i n}=\frac{1000 \mathrm{~W}}{60 v_{i n} S}
$$

Among them, $W$ is the amount of powder given per unit time $(\mathrm{g} / \mathrm{min}), V_{\text {in }}$ is the inlet velocity of airflow $(\mathrm{m} / \mathrm{s})$, which can be measured by anemometer, and $S$ is the cross-sectional area of airflow $\left(\mathrm{m}^{2}\right)$. Outlet concentration $C_{\text {out }}$ can be measured by the HC-9001 smoke and dust automatic tester according to the principle of constant velocity sampling of particulate matter [20]. The efficiency of the dust collector exceeds $99.5 \%$ by calculation. 


\section{$4 \quad$ Experimental analysis}

Fig. 12. is the new type of solar glass window designed in this paper. Its overall size is $37 \mathrm{~cm} * 30 \mathrm{~cm}$.

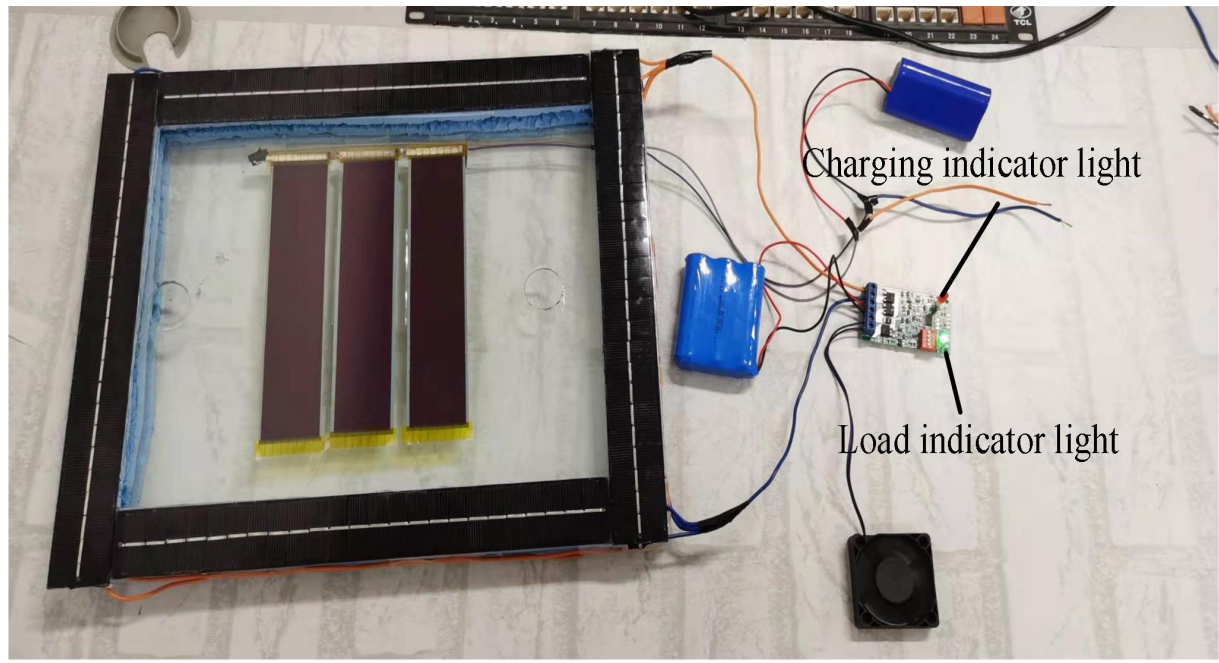

Fig. 12. Physical view of new solar glass Windows

After testing, when the glass window is not placed in the sun and the sun is not enough, the solar controller selects the normally open mode. At this time, the charging indicator light is off but the load indicator light is on, and the battery supplies power for the small fan, as shown in Fig. 13. Show.

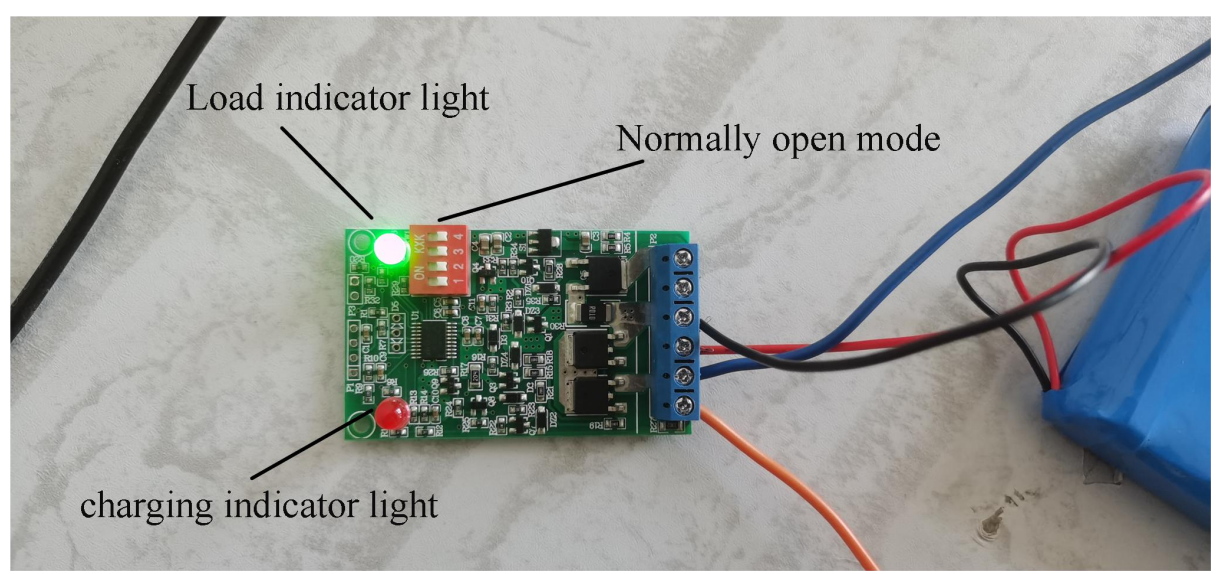

Fig. 13. Diagram of the light on when the light is insufficient

When it is placed in the sun and the sunlight is sufficient, the charging indicator and load indicator will all light up. The solar panel and flexible thin-film solar cell 
generate electricity to charge the battery. The battery can drive a small DC $12 \mathrm{~V}$ fan, as shown in Fig. 14. Show.

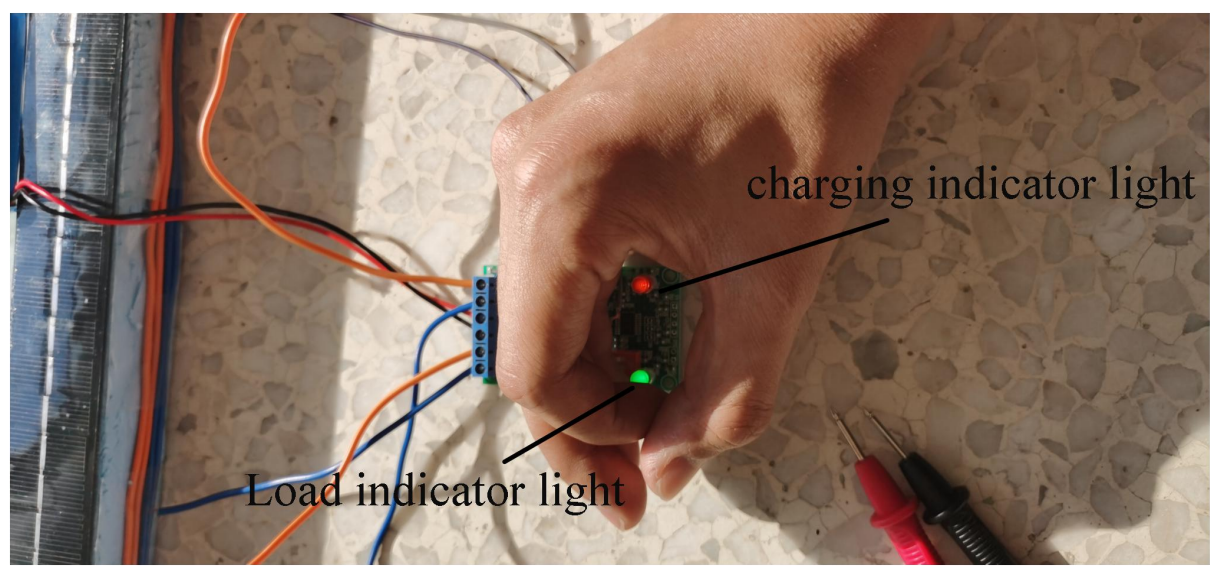

Fig. 14. Picture of the indicator light is on when the light is sufficient

The specific performance and value are shown in Table 1.

Table 1. New solar glass window performance table

\begin{tabular}{lc}
\hline Specific performance & Specific numerical \\
\hline The voltage produced by the solar panel & $11.49 \mathrm{~V}$ \\
The voltage produced by a flexible thin film solar cell & $3.88 \mathrm{~V}$ \\
The storage capacity of an accumulator (connected to a solar panel) & $2000 \mathrm{mAh}$ \\
Storage capacity of a battery (connected to a flexible thin film solar & $6000 \mathrm{mAh}$ \\
cell) & $8.3 \%$ \\
Improve the energy saving effect & $99.5 \%$ \\
Dust removal efficiency & $1.91 \%$ \\
Photoelectric conversion efficiency &
\end{tabular}

\section{5 conclusion}

In this paper, aiming at the problems such as excessive indoor temperature in hot summer and waste of electricity caused by the use of air-conditioning and other cooling equipment, a new type of solar glass window with photovoltaic power generation and intelligent control of indoor temperature is designed. Flexible thin-film solar cells combined with outer glass and solar panels mounted on window frames can generate electricity for indoor air conditioning, according to the temperature sensor intelligent control of the air conditioning on and off, to solve the problem of waste of air conditioning energy consumption, at the same time, in order to improve the photoelectric conversion efficiency of the whole system and reduce the influence of ash accumulation on the solar cells, an automatic dust removal device is installed on 
the outside of the glass window, and the solar cells are cleaned automatically according to the photoelectric sensor. The new solar glass window designed in this paper is of great significance to indoor cooling and energy saving.

\section{References}

1. Wang Jinpeng., Ye Zhengpeng., Jeremy Gillbanks., Tarun M. Sanders and Zou Nianyu.: A Power Control Algorithm Based on Chicken Game Theory in Multi-Hop Networks. Symmetry 2019, 11(5),718.

2. Zhang Ying.: Research on the Application of Solar Energy in Rural Residential Buildings in the Sichuan Basin[D]. Southwest Jiaotong University, 2018.

3. Li Kai., Zhang Xianmin., Zhang Xiang.: Optimum design of front electrode grid line of non-equal width main grid of solar cell[J]. Acta Solar Energy,2021,42(01):69-76.

4. Singh S., Agrawal S.: Efficiency maximization and performance evaluation of hybrid dual channel semitransparent photovoltaic thermal module using fuzzyfied genetic algorithm [J]. Energy Conversion \& Management, 2016, 122: 449-461.

5. Gaur A., Tiwari G N., Ménézo C., et al.: Numerical and experimental studies on a Building integrated Semi-transparent Photovoltaic Thermal(BiSPVT)system: Model validation with a prototype test setup [J]. Energy Conversion \& Management, 2016, 129 : 329-343.

6. Huang Ye., Zhang Gaoming., Gao feng., Liu Zhibing., Wang Zexin., Wei Jinjia.: The thermoelectric performance of dual-axis tracking and single-axis north-south tracking CPV/T system[J]. Solar Energy,2021(02):66-73.

7. Xiaoli Wu.: Influence of temperature of photovoltaic insulating glass modules on electrical performance [D]. Xi 'an University of Technology,2013.

8. Wang Jinpeng., Cao Fan., Zou Nianyu.: Multi carrier system joint receiving method based on MAI and ICI. [J]. Jilin Daxue Xuebao (Gongxueban), 2018, 41(6): 301-305.

9. Wang Jinpeng., Cao Fan., Zou Nianyu.: Cooperative distributed antenna transmission method based on co-channel interference in $5 \mathrm{G}$ mobile communication system[J]. Jilin Daxue Xuebao (Gongxueban),2020, 48 (1) : 333-341.

10. Jun Gao., Hongyun Wang.: Application research of Photovoltaic power generation System in building Energy Saving [J]. Equipment Maintenance Technology,2020(02):64.

11. Wang Jinpeng., Ye Zhengpeng., Tarun M., Bo Li and Zou Nianyu.: A Novel Linear Antenna Synthesis for Linear Dispersion Codes Based on an Innovative HYBRID Genetic Algorithm. Symmetry 2019, 11(9), 1176.

12. Chen Chang.: research on the application of solar photovoltaic ventilated glass Windows in architecture based on CFD simulation [D]. Inner Mongolia: Inner Mongolia University of Technology. 2015, 1-63.

13. Lan Yu., Jie Zhang., Xiaoman Zhai., Qiming Zhao., Huijie Guo.: Design of intelligent Ventilation System powered by Solar Energy [J]. Science and Technology Economy Guide. 2017, (18) : 107.

14. Su Jun., Lin Yangfan., Yu Guanxia., Luo Min.: Thermistor characteristics and the design of temperature control experimental instrument[J]. Physics Experiment of College, 2019, 32(05): 60-63.

15. Yu Duan.: Research on the function and progress of battery modules in photovoltaic power generation unit system [J]. Smart city,2019, 5(20): 80-81.

16. Tao Wang.: Principle and Application of Photoelectric Sensor [J]. Computer Products and Circulation, 2018(07): 64+122. 
17. Jinpeng Wang., Zhengpeng Ye., Fan Cao., Nianyu Zou.: Collaborative Distributed Antenna Transmission System based on Co-frequency Interference distribution in 5G Mobile Communication [J]. Journal of jilin university (engineering science), 2020, 50(01): 333-341.

18. Wang Jinpeng., Cao Fan., Zou Nianyu.: Cooperative distributed antenna transmission method based on co-channel interference in $5 \mathrm{G}$ mobile communication system[J]. Jilin Daxue Xuebao (Gongxueban), 2020, 48(1): 333-341.

19. Shoufeng Zhang., Xiaoyan Liu.: Design of Photovoltaic Dust removal Detection System based on MSP430 [J]. Information Technology and Informatization, 2017(11): 17-19.

20. Zhenmin Yang., Chao Huang., Jiahao Wang., Wenting Geng.: Surfactant influence on wet electrostatic precipitator performance [J/OL]. Environmental engineering: 1-10 (2020). http://kns.cnki.net/kcms/detail/11.2097.X.20201124.1749.010.html. 